\title{
Redes sociales, acción colectiva y elecciones: Ios usos de Facebook por el movimiento estudiantil chileno durante la campaña electoral de 2013
}

\section{Lázaro M. Bacallao-Pino'}

Recibido: 2015-07-16

Enviado a pares: 2015-07-19
Aprobado por pares: 2015-11-18

Aceptado: 2016-01-02

DOI: 10.5294/pacla.2016.19.3.6

Para citar este artículo / to reference this article / para citar este artigo

Bacallao-Pino, L. M. (2016). Redes sociales, acción colectiva y elecciones: Ios usos de Facebook por el movimiento estudiantil chileno durante la campaña electoral de 2013.

Palabra Clave 19(3), 810-837. DOI: 10.5294/pacla.2016.19.3.6

\section{Resumen}

Los estudios recientes sobre las redes sociales digitales han analizado, por un lado, sus usos como parte de la acción colectiva y, por otro, sus usos durante las campañas electorales. Basado en el estudio del movimiento estudiantil chileno durante la campaña electoral de 2013 en ese país, el texto analiza los usos de Facebook por parte de tres federaciones estudiantiles que integran dicho movimiento. Los resultados indican que se priorizan las dimensiones informativa y organizativa en los usos de las redes sociales en ese contexto. Esta tendencia está mediada por las particularidades del movimiento - presencia de organizaciones estudiantiles tradicionales- y por la complejidad de la relación con la política institucional —en particular, la defensa de la autonomía frente a los riesgos de cooptación, acrecen-

1 Universidad de Chile, Chile. lazaro_bacallao@biari.brown.edu 
tados por el tránsito de exlíderes del movimiento a esta última-. De igual forma, también se confirma la tendencia a un mayor uso de estos espacios en momentos de mayor movilización social y se muestran las tensiones entre sus usos por parte de la acción colectiva organizada (federaciones estudiantiles) y los sujetos individuales participantes en ella.

\section{Palabras clave}

Movimiento estudiantil; Chile; redes sociales; elecciones; TIC (Fuente: Tesauro de la Unesco). 


\section{Social Networks, Collective Action and Elections: How the Chilean Student Movement Used Facebook during the 2013 Electoral Campaign}

\section{Abstract}

Recent studies on digital social networks have analyzed their role as part of collective action and their use during electoral campaigns. Based on a study of the Chilean student movement during the 2013 electoral campaign in that country, the article offers an analysis of how Facebook is used by the three student federations that make up the movement. The results suggest informative and organizational aspects take priority in the use of social networks in that context. This trend is mediated by the peculiarities of the movement - the presence of traditional student organizations - and by and the complexity of the relationship with institutional policy, particularly the defense of autonomy against the risks of cooptation, increased by the passage of former leaders of the movement to the latter. The inclination for these spaces to be used more and more at times of increased social mobilization is confirmed, and the tensions between their use by organized collective action (student federations) and by the individuals participating in that action are shown.

\section{Keywords}

Student movement; Chile; social networks; elections; ICT (Source: Unesco Thesaurus). 


\section{Redes sociais, ação coletiva e eleições: os usos do Facebook pelo movimento estudantil chileno durante a campanha eleitoral de 2013}

\section{Resumo}

Os estudos recentes sobre as redes sociais digitais têm analisado, por um lado, seus usos como parte da ação coletiva e, por outro, seus usos durante as campanhas eleitorais. Baseado no estudo do movimento estudantil chileno durante a campanha eleitoral de 2013 nesse país, o texto analisa os usos do Facebook pelas três federações estudantis que integram esse movimento. Os resultados indicam que são priorizadas as dimensões informativa e organizativa nos usos das redes sociais nesse contexto. Essa tendência está intermediada pelas particularidades do movimento — presença de organizações estudantis tradicionais - e pela complexidade da relação com a política institucional —em particular, a defesa da autonomia ante os riscos de cooptação, acrescentados pelo trânsito de ex-líderes do movimento a esta última- Da mesma forma, também se confirma a tendência a um maior uso desses espaços em momentos de maior mobilização social e mostramse as tensões entre seus usos por parte da ação coletiva organizada (federações estudantis) e os sujeitos individuais participantes dela.

\section{Palavras-chave}

Movimento estudantil; Chile; redes sociais; eleições; TIC (Fonte: Tesauro da Unesco). 


\section{Introducción}

Las redes sociales digitales han devenido recursos tecnológicos relevantes en las sociedades contemporáneas, en particular entre los jóvenes (Duggan et al., 2014). Su relevancia para la acción colectiva o las dinámicas políticas institucionales se inserta en el amplio debate acerca del impacto de las tecnologías de la información y la comunicación (TIC) en la política tradicional (Dahlberg y Siapera, 2007), como las campañas electorales (Davis, Elin y Reeher, 2002), o sobre su potencial para configurar una nueva era de democracia participativa (Dahlgren, 2005).

Por una parte, el uso de las redes sociales es una dimensión central en los estudios acerca de las movilizaciones sociales, desde el 15M en España, Occupy Wall Street o el \#YoSoy132 en México (Juris, 2012; Cerrillo y Lay, 2014; Anduiza, Cristancho y Sabucedo, 2014). Al mismo tiempo, también se ha analizado su mediación en la política institucional, en particular durante las campañas electorales (Panagopoulos et al., 2009; Vitak et al., 2011; Sarıtaş y Aydın, 2015).

Sin embargo, hasta donde sabemos, no hay estudios previos en los cuales se articulen ambas dimensiones analíticas, a partir del examen de los usos de las redes sociales por parte de movimientos sociales ya existentes, en el contexto campañas electorales. ${ }^{2}$ Desde esta perspectiva, el análisis de tal cuestión en el movimiento estudiantil chileno durante las elecciones de 2013 en ese país resulta un caso analíticamente significativo.

En primer lugar, por el carácter juvenil de este movimiento y el alto uso de las redes sociales por los usuarios de internet de entre 18 y 29 años (Duggan et al.,2014). Las investigaciones previas han mostrado que el movimiento estudiantil chileno ha hecho un importante uso de las redes sociales, en particular de Facebook, durante sus movilizaciones sociales (Cabalín-Quijada, 2014; Valenzuela, Arriagada y Scherman, 2012). Además, es relevante por la peculiar relación entre movimiento estudiantil y política institucional chilena, en especial el tránsito hacia esta última, precisamente durante

2 Se excluye aquí el caso del movimiento mexicano \#YoSoy132, dado que surgió precisamente en el contexto de la propia campaña electoral de 2012 en ese país, por lo que no era un movimiento existente previamente. 
la campaña electoral de 2013, de varios de los principales líderes del movimiento. Ello es significativo, pues la relación entre movimientos sociales y política institucional — en particular, partidos políticos — ha sido una dimensión en especial polémica en las teorizaciones acerca de estos agentes de la acción colectiva (Routledge, 1997; Offe, 1988).

Varios de esos exdirigentes del movimiento estudiantil fueron candidatos a diputados al Congreso por distintas formaciones políticas, integrantes o no de la Nueva Mayoría, la colación de centroizquierda liderada por Michelle Bachelet. Por ejemplo, Camila Vallejo, presidenta de la Federación de Estudiantes de la Universidad de Chile (FECH) en 2010-2011; Giorgio Jackson, presidente de la Federación de Estudiantes de la Universidad Católica (FEUC) en 2011, y Gabriel Boric, presidente de la FECH en 2012. Otros exdirigentes formaron parte del comando electoral de la candidata Bachelet, como es el caso de Camilo Ballesteros, presidente de la Federación de Estudiantes de la Universidad de Santiago de Chile (Feusach) en 2010-2011.

La complementación de ambas circunstancias configura un contexto analítico relevante para el examen de las interrelaciones entre movimientos sociales y política institucional, a partir del uso de las redes sociales por parte de los primeros en un momento crítico para la segunda (campañas electorales). A ello se añade la particularidad del contexto político de las elecciones generales chilenas de 2013, marcadas por 1) el enfrentamiento en las urnas al primer gobierno de derechas luego del retorno a la democracia, liderado por Sebastián Piñera; 2) la apertura de la Concertación y su conversión en la compleja Nueva Mayoría, con la inclusión de nuevos partidos de izquierda en la coalición, entre ellos el Partido Comunista de Chile (PCCh), la Izquierda Ciudadana (IC) y el Movimiento Amplio SocialRegión (MAS Región). En este escenario electoral, coagulan "el complejo campo de interacciones establecidas entre este actor social [el movimiento estudiantil] y el gobierno nacional durante el período 2006-2013”, tras lo cual se abrió una serie de interrogantes acerca de "la necesidad de repensar la relación con el Estado, su institucionalidad y los partidos políticos” y, de hecho, su tránsito "de la arena social a la arena política” (Paz, 2014, p. 102). 


\section{Metodología: caso de estudio, métodos y técnicas}

El objetivo del artículo es analizar los usos de Facebook por parte del movimiento estudiantil chileno durante la campaña electoral de 2013 en Chile, entre el 17 de octubre y el 18 de diciembre de 2013. Se han seleccionado los perfiles en esta red social de las federaciones estudiantiles de tres importantes universidades tradicionales chilenas, ${ }^{3}$ dos públicas: la Universidad de Chile (UCh) y la Universidad de Santiago de Chile (Usach), y una privada: la Pontificia Universidad Católica de Chile (UC). El perfil de la FEUC fue creado en 2011, y actualmente cuenta con 18104 seguidores. También creado ese mismo año, el de la Feusach tiene 28708 seguidores, mientras que el de la FECH, creado en 2009, cuenta con 96324 seguidores. Tanto en los perfiles de la Fuesach como de la FECH el número de seguidores supera el total de alumnos matriculados en las respectivas instituciones, alcanzando en el segundo caso 2.5 veces el total alumnos de la Universidad, mostrando la relevancia comunicativa de la tales instituciones en esta red social en Chile.

Esta selección se explica, en primer lugar, porque los líderes de sus respectivas federaciones estuvieron entre quienes tuvieron una mayor visibilidad durante las movilizaciones de 2011 y que, en las elecciones de 2013, pasaron a la política institucional. Además, a diferencia de otros episodios recientes de movilización social, no existe un perfil de Facebook específico del movimiento estudiantil chileno, por lo que las investigaciones previas también han optado por analizar los espacios en estas plataformas de las organizaciones estudiantiles (Cabalín-Quijada, 2014). Ello es coherente con la importancia de las federaciones estudiantiles para este movimiento social. A diferencia de otros episodios recientes de acción colectiva, como el 15M, Occupy Wall Street o el \#YoSoy132, en el movimiento estudiantil chileno, las protestas no han tenido lugar a través de pequeños grupos coordinados por redes débiles, sino impulsadas por estas organizaciones estudiantiles, lo cual remite a las formas clásicas de la política de la protesta (Guzmán-Concha, 2012). La importancia de estas organizaciones en el caso chileno también ha sido subrayado por Castells (2014a), al compa-

3 Las universidades tradicionales son aquellas que integran el Consejo de Rectores de las Universidades Chilenas (CRUCH). 
rar el movimiento estudiantil chileno con otras movilizaciones recientes, como las antes mencionadas, caracterizadas por emerger sin líderes ni organización y al margen de partidos políticos y sindicatos, mientras que en el caso chileno los líderes del movimiento aparecen vinculados a partidos y organizaciones políticas, con militancias previas en el PCCh (Camila Vallejo, Camilo Ballesteros), o la Izquierda Autónoma (Gabriel Boric, Francisco Figueroa), así como la participación fundacional en nuevas organizaciones políticas posteriores a las movilizaciones de 2011 (como es el caso de Giorgio Jackson, fundador de Revolución Democrática en 2012).

Desde una perspectiva metodológica que integra la mirada cuantitativa y la cualitativa, se ha realizado el seguimiento de los perfiles en Facebook de la FECH, la FEUC y la Feusach en el periodo indicado, abarcando así los dos momentos de la campaña electoral (primera y segunda vuelta). El inicio del seguimiento coincide con la última gran marcha nacional de 2013, convocada para el día previo al comienzo de la campaña por la Confederación de Estudiantes de Chile (Confech), y termina tres días después de la celebración de la votación en la segunda vuelta. En primer lugar, se han contabilizado el total de posts, así como los me gusta, las veces en que fueron compartidos y los comentarios a estos. Con tales datos, se han calculado las respectivas medias de cada recurso interactivo durante el periodo, en general, y para los posts específicamente relacionados con la campaña electoral. Se ha calculado la tasa de interactividad $\left(\mathrm{T}_{\mathrm{i}}\right)$ de cada perfil de Facebook, siguiendo la siguiente fórmula de media ponderada /100: $\mathrm{T}_{\mathrm{i}}=\left(\mathrm{M}_{\mathrm{mg}}\right.$ $\left.{ }^{*} 0.25\right)+\left(\mathrm{M}_{\mathrm{cp}}{ }^{*} 0.25\right)+\left(\mathrm{M}_{\mathrm{co}}{ }^{*} 0.50\right) / 100$, donde $\mathrm{M}_{\mathrm{mg}}$ se refiere a la media de los me gusta, $\mathrm{M}_{\mathrm{cp}}$, a la media de las veces en que se compartieron los posts y $\mathrm{M}_{\mathrm{co}}$ a la de los comentarios.

Esta fórmula — utilizada en estudios previos realizados por el autorpermite dar un mayor peso a los comentarios como expresión de interactividad en Facebook ( $50 \%$ ), en comparación con los me gusta y compartir ( $25 \%$ a cada uno), pues los comentarios tienen una mayor carga de sentidos, ya que también pueden ser analizados cualitativamente. La métrica personas hablando de esto, propia de Facebook, no puede ser empleada en este caso, pues toma como referencia los siete días previos al momento en que 
se realiza la observación. La ponderación propuesta en la fórmula es coherente con estudios anteriores, los cuales asocian la interactividad en Facebook a la generación de un diálogo tanto entre el administrador del perfil y los usuarios como entre estos últimos, destacando precisamente que tales "conversaciones pueden darse por medio de comentarios, en un grado más alto, y a través de 'me gusta' o 'compartir', en grados inferiores” (Zonta, 2013, p. 65). Otros análisis solo contabilizan los comentarios y me gusta, otorgando el mismo peso a ambos en el análisis del porcentaje de usuarios - con respecto al total de seguidores - que comentan o indican que les gusta un post determinado publicado en el perfil de la red (Túñez, 2012, pp. 234-235).

En segundo lugar, se ha procedido al análisis del discurso de aquellos posts y comentarios vinculados a la campaña electoral de otros textos propios del movimiento publicados durante la campaña (declaraciones, manifiestos, etc.), así como a los discursos obtenidos en doce entrevistas realizadas con dirigentes del movimiento estudiantil chileno tanto de 2011 —incluidos algunos de los que transitaron hacia la política institucionalcomo de 2013. Las entrevistas en profundidad se realizaron en Santiago de Chile, entre enero y abril de 2015, y los entrevistados incluyeron a todos aquellos líderes del movimiento estudiantil que accedieron a participar en el estudio. Si bien la disponibilidad de los entrevistados puede considerarse un sesgo, se debe tener en cuenta que la dimensión cualitativa no tiene un propósito de representatividad en términos estadísticos, sobre todo al tratarse de un estudio de caso. Además, se ha logrado entrevistar a dirigentes del movimiento estudiantil de las tres federaciones incluidas en el estudio y de las principales tendencias políticas presentes en el movimiento estudiantil: comunismo, izquierda autónoma y socialdemocracia.

El análisis de discurso se ha convertido en un importante método para la investigación en movimientos sociales (Taylor y Whittier, 2004), en particular en relación con los procesos de construcción de sentido (Melucci, 2004). Los estudios precedentes han aplicado distintos enfoques del análisis del discurso en la investigación sobre las redes sociales digitales, como el análisis del discurso mediado por computadoras propuesto por Herring (2004) o el análisis crítico del discurso de Fairclough (1995). 
Este último se adecua a los objetivos planteados, pues proporciona un marco analítico para desvelar las relaciones entre discurso y sociedad, a través del examen de la elección de ciertas palabras y metáforas por parte de los participantes en el discurso (Fairclough, 1995), sobre la base de una comprensión de este en cuanto faceta de la vida social en interacción con otras dimensiones sociales y, en última instancia, como práctica social (Fairclough, 2003). En tal sentido, Franzosi (1998) propone superar enfoques restrictivos del análisis del discurso para incluir otras dimensiones discursivas, como la estructura y secuencias narrativas de los textos, o las propias relaciones sociales e interrogantes en torno a estas. El análisis se ha centrado en tales dimensiones y su presencia en los perfiles de Facebook (posts y comentarios), en otros textos vinculados al movimiento (declaraciones, etc.) y en los obtenidos en las entrevistas. En particular, nos hemos focalizado en 1) valoraciones sobre la presencia de las demandas del movimiento en la agenda electoral; 2) la expresión de las interrelaciones entre movimiento social y política institucional en el contexto de la campaña, y 3) dimensiones $y$ aspectos que median esas interrelaciones.

\section{Estado de la cuestión}

Las relaciones entre movimientos sociales y política institucional han sido objeto de atención desde distintas propuestas teóricas. Al respecto, Offe (1998) plantea tres posibilidades: 1) las demandas y reivindicaciones centrales de los primeros pueden ser satisfechas y, en consecuencia, estos pierden "mérito", 2) la agudización del conflicto, que da lugar a situaciones de alta represión elevadoras de los costes de la movilización y 3 ) un posible proceso de "institucionalización" del movimiento social. En esta última, se articulan dos aspectos: 1) la transformación del movimiento social en partido para reformar "desde dentro" la política institucional y 2) el predominio de las formas de presión y negociación en el ámbito de los procedimientos institucionales estatales.

Routledge (1997) identifica tres posibles respuestas del Estado ante los movimientos sociales: la coacción (represión), la cooptación y la mediación, o una mezcla de las tres. Frente a ello, los movimientos sociales asumen como uno de sus principios fundamentales la autonomía, proponiéndose 
gestar procesos de cambio social en sus experiencias, lejos de los espacios sociopolíticos tradicionales (Zibechi, 2004). Pero, a pesar de esta autonomía, al mismo tiempo se reconoce la existencia de una relación directa entre los movimientos sociales en red y los procesos de transformación en el sistema político, a partir de la inclusión de las demandas de los primeros en la agenda de los segundos (Castells, 2014). Precisamente, en la sociedad red, en cuanto estructura social de la sociedad red caracterizada por "la presencia ubicua de redes de comunicación en un hipertexto modal”, los movimientos sociales, a través de la autocomunicación de masas basada en su uso de esas redes, cuestionan las relaciones de poder institucionalizadas, de forma tal que "la transformación de la comunicación de masas en autocomunicación de masas ha contribuido de forma decisiva a modificar el proceso del cambio social", pues "la proliferación de redes horizontales de comunicación ha generado un nuevo paisaje de cambio social y político, a través de un proceso de desintermediación de los controles gubernamentales y corporativos sobre las comunicaciones" (Castells, 2014b, p. 144).

La autocomunicación de masas basada en las tecnologías de la información y de la comunicación (TIC), y que tiene en los movimientos sociales a uno de sus actores fundamentales, ha configurado un nuevo entorno comunicativo (Castells, 2009, 2012), de forma que el poder de la red reside en la posibilidad de que "los actores sociales construyen sus propias redes según sus propios proyectos, valores e intereses” (Castells, 2014b, p. 144).

La comunicación es, por tanto, una dimensión clave en la comprensión de las interrelaciones de los movimientos sociales con la política institucional. Un momento de singular relevancia, en tal sentido, son las campañas electorales, cuando la comunicación política presenta características específicas, con lógicas diferentes en lo que se refiere al comportamiento de los actores políticos, su reacción frente a la cobertura mediática y las propias dinámicas de esta última (Walgrave y Van Aelst, 2006). En este contexto, los análisis de las interrelaciones entre partidos y movimientos sociales se han focalizado precisamente en la dimensión comunicativa: la comparación entre medios tradicionales y espacios digitales (Escandón, 2013) o la creación de canales propios por los participantes en la acción colectiva 
durante el proceso electoral (Fernández-Planells, Feixa Pampols y Figueroas-Maz, 2013).

Sin embargo, exceptuando los estudios sobre las movilizaciones sociales surgidas en contextos de campañas electorales - como el 15M en España, configurado durante la campaña para las elecciones autonómicas de 2011, y el \#YoSoy132 en México, nacido en el entorno de la campaña para las elecciones presidenciales de 2012-, la indagación en torno a los usos de las redes sociales por parte de los movimientos sociales y por los partidos políticos durante las campañas electorales, ha transitado caminos separados.

Por un lado, los estudios previos han analizado los usos de estas plataformas de la Web 2.0 como parte de la acción colectiva, considerándolas una importante herramienta en la creación, gestión y difusión de la protesta social (Castillo, García y Smolak, 2013). Se ha destacado su impacto en la dimensión organizativa mediante la emergencia de una "lógica de la acción conectiva, basada en la contenidos personalizados compartidos a través de las redes digitales" (Bennet y Segerberg, 2012, p. 739), pero también en el impulso de procesos significativos en la cultura política, la democratización mediática y un mayor pluralismo (Gómez y Treré, 2014). En el movimiento estudiantil chileno, las investigaciones anteriores han destacado el uso de las redes sociales durante las movilizaciones con fines informativos y de socialización, como fuente de información, así como para movilizar a simpatizantes, destacar logros y señalar a sus oponentes (Valenzuela, Arriagada y Scherman, 2012; Cabalín-Quijada, 2014).

Por otra parte, el uso de las redes sociales durante las campañas electorales tanto por los ciudadanos en general como por los partidos políticos y los medios ha devenido una línea de investigación de creciente importancia. En este escenario, las redes sociales son usadas por los ciudadanos para compartir creencias políticas, apoyar candidatos e interactuar con otros sujetos sobre temas políticos (Vitak et al., 2011). Se ha señalado que permiten a los individuos acceder a cualquier tipo de información — parcial o imparcial - y recibir una retroalimentación rápida y transparente, ofreciendo la posibilidad a los candidatos, políticos y partidos de una mayor 
cercanía con los electores (Sarıtaş y Aydın, 2015). En particular, Facebook es considerado una herramienta viable para la comunicación política durante campañas electorales, mediante la creación de grupos con una elevada actividad, como la campaña presidencial de 2008 en los Estados Unidos (Woolley, Limperos y Oliver, 2010). Este mismo caso ha servido para subrayar las oportunidades ofrecidas por Twitter para las campañas digitales y la deliberación en línea (Larsson y Moe, 2012). Los estudios previos sobre las interrelaciones entre agentes mediáticos, políticos y ciudadanos a través de Twitter durante campañas electorales han subrayado la permanencia de la centralidad de los dos primeros en los patrones conversacionales, como resultado del elevado número de menciones y de la cercana conexión entre ambos (D'heer y Verdegem, 2014).

La articulación entre ambos usos de las redes sociales - por un lado, por los movimientos sociales, y por otro, durante campañas electoralesresulta, por consiguiente, relevante, con el objetivo de identificar las posibles dis-continuidades en las tendencias identificadas por los estudios precedentes sobre cada una de estas dos líneas de indagación. Este análisis permitirá profundizar, desde la dimensión comunicativa, en las interrelaciones entre acción colectiva y política institucional en un entorno relevante, como son las campañas electorales.

Tomar como referencia para el análisis el caso del movimiento estudiantil chileno en el ámbito de la campaña electoral de 2013 hace posible examinar la posible continuidad y sedimentación (o no) de las tendencias en los usos de las redes sociales identificadas por estudios anteriores durante los periodos de mayor auge de la acción colectiva (Valenzuela, Arriagada y Scherman, 2012; Cabalín-Quijada, 2014), en contexto caracterizado por 1) el predominio de la comunicación política institucional (campaña electoral); 2) un menor grado de movilización social en comparación con otros momentos, como 2011 ; 3) la incorporación a la política institucional de varios exlíderes del movimiento, y 4) la inclusión de las demandas del movimiento estudiantil en las agendas electorales, sobre todo de la oposición (Nueva Mayoría), liderada por Bachelet. En tal contexto, se plantea como premisa que los usos de las redes sociales —en particular, Facebook — por parte 
del movimiento estarán mediados por ese escenario y presentará tendencias específicas en comparación con los periodos de mayor movilización social.

\section{Resultados}

En el período analizado, se publicaron 126 posts en el perfil de $\mathrm{FECH}, 99$ en el de la FEUC y 108 en el de la Feusach, para una media de 2.00, 1.57 y 1.71 posts por día, respectivamente. En el perfil de la $\mathrm{FECH}$, se registró un promedio de 19.03 me gusta, 3.54 compartir y 1.28 comentarios por cada post; en el perfil de la FEUC en la red social, estas cifras fueron de 17.17, 0.79 y 0.59 , respectivamente, mientras que en el perfil de la Feusach, los promedios fueron de 10.18, 2.94 y 1.43, en cada uno de estos recursos de interactividad.

Estos datos muestran que los me gusta destacan por una media significamente mayor en comparación con los compartir y los comentarios. El uso los dos recursos de interactividad que más pueden ser asociados a un sentido de lo colectivo - el hecho de compartir algo o de participar en un debate- resulta muy bajo en comparación con aquel recurso que tiene una proyección más individual, el hecho de declarar que algo gusta.

La tasa de interactividad durante el periodo analizado fue de 0.06 en el perfil de la FECH, 0.05 en el de FEUC y 0.04 en el de la Feusach, valores que se corresponden con los bajos niveles de uso de los recursos de interactividad disponibles en esta red social, en particular de los comentarios. Estos valores resultan significativamente bajos cuando se comparan con la tasa de interactividad observada en estudios previos en el uso de Facebook por otros movimientos sociales. En cuanto a la utilización de recursos, como la creación de eventos o la realización de encuestas a través de Facebook, durante los tres meses analizados, en el perfil de la FECH se crearon cinco eventos con una media de 122.8 participantes; en la FEUC, cuatro eventos, con un promedio de 669 participantes y 10 eventos en el perfil de la Feusach, con una media de 173.3 participantes.

El comportamiento, a lo largo del periodo analizado, del número de posts publicados en cada uno de los tres perfiles se muestra en la figura 1 . 
El análisis de las tendencias muestra que el incremento de la actividad no se corresponde con momentos relevantes de la campaña, como los debates realizados entre candidatos $(25,29$ y 30 de octubre de 2013; 6 y 10 de diciembre de 2013) o las votaciones (17 de noviembre y 15 de diciembre de 2013). En cambio, sí hay una mayor actividad — mayor número de posts - durante la realización de acciones colectivas (marchas, manifestaciones, etc.), así como en el contexto de procesos internos importantes para las respectivas federaciones estudiantiles.

Figura 1. Número de posts publicados en cada perfil de Facebook entre el 17 de octubre y el 18 de diciembre de 2013

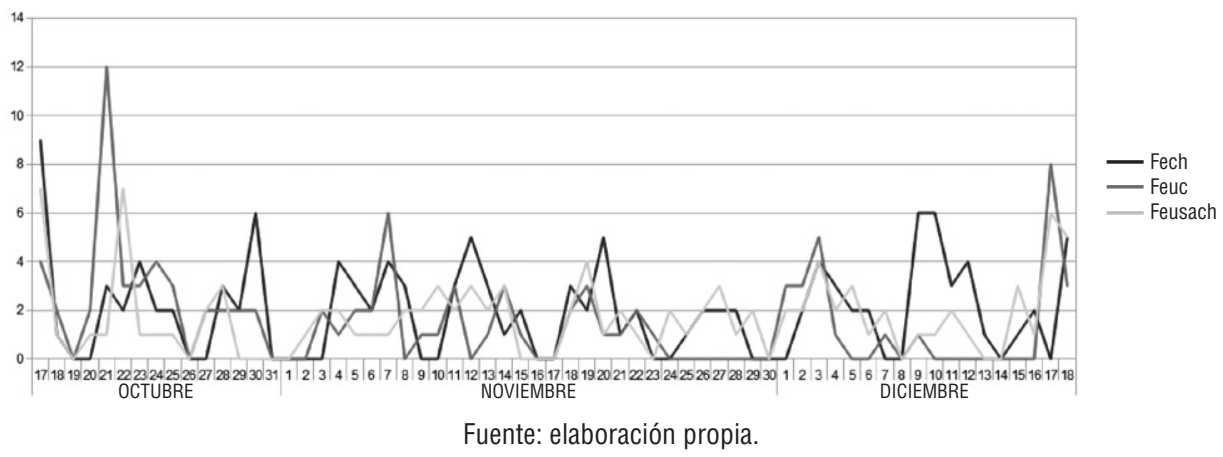

Ejemplo de lo primero es el incremento de posts el 17 de octubre, cuando se celebró la última marcha nacional convocada por la Confech, con el propósito de "forzar a las candidaturas a definirse en función del petitorio del Movimiento Social por la Educación, que ya había consolidado respaldo y solidaridad por parte de la ciudadanía” (FA, FECH, Santiago, abril de 2015) $)^{4}$ "reivindicar en este año de elecciones las demandas que han venido saliendo desde 2011" (comentario, perfil de la FECH, 17 de octubre). También el mayor número de posts el 9 y 10 de diciembre en el perfil de la $\mathrm{FECH}$, asociado a la realización de una concentración contra el alza de los aranceles en la Universidad de Chile; el 17 de diciembre en el caso del perfil de la Feusach, vinculado a una marcha en la Universidad de Santiago de Chile para visibilizar las demandas internas, como la democrati-

4 Los discursos de los entrevistados serán identificados de esta forma: iniciales del entrevistado, organización estudiantil de pertenencia, lugar y fecha de la entrevista. 
zación y la estabilidad laboral; o también el 17 de diciembre en el perfil de la FEUC, como parte de la convocatoria a una celebración navideña colectiva. El número de posts también se incrementa en momentos vinculados a procesos internos en las organizaciones estudiantiles, como los respectivos procesos electorales internos de las federaciones: la FEUC ( 21 de octubre y 7 de noviembre) y la FECH (30 de octubre y 12 de noviembre).

En los posts publicados en los perfiles de las tres organizaciones estudiantiles, hay una baja presencia de la cuestión electoral en el contexto de la campaña. Como muestra la tabla 1 , el número de posts acerca del tema apenas alcanza $0.93 \%$ en la Feusach — si se incluye un evento en el cual se llevó a cabo un análisis de la franja electoral en los medios, por parte de expertos, este porcentaje se elevaría a $1.85 \%$-; es $1.01 \%$ en la FEUC y $3.97 \%$ en la FECH. La tasa de interactividad específica de estos posts fue de 0.04 en la FEUC, 0.001 en la Feusach y 0.10 en el perfil de la FECH, lo cual muestra una tendencia al incremento de la tasa de interactividad en este último — si bien sigue siendo baja, casi se duplica - y una disminución en los dos primeros.

Tabla 1. Número de posts publicados en cada perfil según los temas y porcentaje que representa en el total de posts publicados en cada uno durante el periodo analizado

\begin{tabular}{|c|c|c|c|c|c|c|}
\hline Tema del post & Fech & $\%$ del total & Feuc & $\%$ del total & Feusach & $\%$ del total \\
\hline Acción colectiva & 21 & 16.67 & 7 & 7.07 & 20 & 18.52 \\
\hline $\begin{array}{l}\text { Compartir contenidos } \\
\text { (vídeos, } \\
\text { informaciones, } \\
\text { imágenes, etc.) }\end{array}$ & 43 & 34.13 & 31 & 31.31 & 69 & 63.89 \\
\hline $\begin{array}{l}\text { Procesos internos del } \\
\text { movimiento }\end{array}$ & 33 & 26.19 & 50 & 50.51 & 4 & 3.7 \\
\hline Elecciones & 5 & 3.97 & 1 & 1.01 & 1 & 0.93 \\
\hline $\begin{array}{l}\text { Contenidos de otros } \\
\text { medios }\end{array}$ & 19 & 15.08 & 5 & 5.05 & 3 & 2.78 \\
\hline El perfil & 0 & 0 & 1 & 1.01 & 1 & 0.93 \\
\hline Creación de evento & 5 & 3.97 & 4 & 4.04 & 10 & 9.26 \\
\hline TOTAL & 126 & & 99 & & 108 & \\
\hline
\end{tabular}


En cambio, hay un importante uso de los perfiles como recursos informativos a partir de publicar/compartir contenidos, con unos porcentajes de posts que en todos los casos superan $30 \%$, y que en el perfil de la Feusach alcanza más de la mitad de los posts publicados en el periodo. Estos datos también muestran la importancia otorgada a esta plataforma de la Web 2.0 como recurso vinculado a los procesos organizativos internos, destacando los elevados porcentajes de posts asociados a este tema en los perfiles de la FECH y la FEUC, las federaciones estudiantiles que llevaron a cabo sus respectivos procesos electorales internos correspondientes a 2014 durante el periodo analizado. También resulta relevante el porcentaje de posts vinculados a la acción colectiva, en particular en las federaciones de las dos universidades públicas incluidas, la de Chile y la de Santiago.

Según los entrevistados, este bajo nivel de posts centrados en la campaña electoral se explica por un uso menos institucional y más espontáneo de las redes sociales:

\begin{abstract}
Si bien desde 2011 nos dimos cuenta de que las redes sociales eran extraordinariamente útiles, porque incluso establecían pautas temáticas y nos permitían contrarrestar la exclusión de la agenda de los medios, este uso no se hacía de forma centralizada y unificada. Es decir, no se hizo un uso de las mismas desde las instituciones oficiales del movimiento sino más bien desde sus agrupaciones más intermedias y de base (FF, FECH, Santiago, enero de 2015).
\end{abstract}

Los perfiles de Facebook analizados están, precisamente, asociados a organizaciones oficiales vinculadas al movimiento, pero, a juicio de los entrevistados, "nunca ha habido un ejercicio unificado, oficial, de las redes sociales” (FF, FECH, Santiago, enero de 2015). El mayor número de posts acerca de los contenidos de los medios tradicionales que se observa se asocia a un uso de estos espacios para lograr visibilidad autónoma frente a los medios, incluso lograr ser puestos en su agenda:

Sí ha habido un marcado interés por llegar a los medios de prensa. [...] Constituimos equipos, preparamos conferencias de prensa, siempre pensando en llegar a los medios tradicionales. Aunque ha habido un discurso crítico de los medios de comunicación, también [...] se ha hecho un esfuerzo enorme para posicionarse en ellos (CB, Feusach, Santiago, enero de 2015). 
La escasa presencia del tema de la campaña también se vincula a una pérdida de capacidad del propio movimiento para mediar en la agenda pública una vez pasado el momento de mayor movilización social de 2011. Se llega a considerar que "no era tanto el movimiento social como las demandas que fueron puestas en agenda por el movimiento, lo que estaba mediando la agenda de la campaña electoral", pues en 2012 y 2013 "no hubo una estrategia ni una táctica clara, de cómo irrumpir comunicativamente" por parte del movimiento (GP, Confech, Santiago, marzo de 2015).

A esta crítica a la falta de una estrategia comunicativa durante la campaña, se añade que, frente al tránsito hacia la política institucional de varios exdirigentes del movimiento, el bajo número de posts sobre la campaña se considera también parte del esfuerzo por

mantener la autonomía del movimiento, frente a la cooptación or-
gánica derivada de la presencia de figuras asociadas al movimiento
en la política tradicional apoyando a una de las candidatas y a la
cooptación simbólica resultado de un desplazamiento del terreno en
disputa y de la asociación directa entre movimiento estudiantil y par-
tidos políticos que disputan el cambio electoral (EG, FECH, Santiago,
abril de 2015).

Es decir que los sujetos asumen el silencio temático como una estrategia de autonomía en unas circunstancias muy complejas para el movimiento, como consecuencia de las candidaturas de varios exlíderes.

Esta cuestión resulta central en el análisis que hacen los entrevistados — sobre todo desde los posicionamientos de la izquierda autónoma-, pues se vincula al debate sobre la dimensión política de los movimientos sociales. Varios entrevistados coinciden en señalar que esta tendencia en la presencia del tema electoral en los perfiles de Facebook está vinculada a "la separación entre lo social y lo político existente en el imaginario de la sociedad chilena” (GP, Confech, Santiago, marzo de 2015). Esta separación provocó que "no tuviésemos una visión clara de nuestra participación en la política institucional en 2013 y hubiera un rechazo casi identitario a la misma, sobre todo en quienes venimos de sectores muy radicales, ideológicamente hablando" (FA, FECH, Santiago, abril de 2015). Al mismo tiempo, esta dicotomía se 
asocia a que "el movimiento priorizara su autonomía y se discutiera mucho en las asambleas que los exlíderes que participaban en la campaña no eran candidatos del movimiento, para evitar la cooptación de lo social por la política institucional” (MS, FECH, Santiago, abril de 2015).

Los posts relacionados con la campaña electoral publicados en los perfiles de Facebook durante el periodo analizado, precisamente visibilizan una relación crítica con los programas de los candidatos presidenciales. En tal sentido, se subraya el papel del movimiento estudiantil en la configuración de la agenda de la campaña, pues "los candidatos, todos, han sido obligados prácticamente a referirse al tema educación”, aunque se critica la forma en que lo han hecho, en particular en el caso de Michelle Bachelet, de quien se afirma que "recoge consignas del movimiento estudiantil, pero no deja la lógica individual” (post, perfil de la FECH, 27 de noviembre).

El pronunciamiento oficial de la Confech sobre las elecciones - reproducido en el perfil de la FECH - destaca la importancia de la acción colectiva durante los años previos para mostrar "las falencias profundas que tiene actualmente nuestro sistema educacional", de las cuales "los últimos gobiernos no han sabido hacerse cargo", como tampoco "ninguna de las candidatas [refiriéndose a las dos que alcanzaron la segunda vuelta] ha podido plantear un programa de gobierno que se haga cargo de manera profunda de las demandas que durante años hemos levantado" (Declaración de la Confech, perfil de la FECH, 13 de diciembre). El propio documento rechaza cualquier manipulación electoralista de la agenda del movimiento: "No queremos ofertones electorales ni una lista de promesas con nuestras consignas: queremos un nuevo modelo educativo".

En tal sentido, a través de estos espacios, se demanda a la candidata ganadora que realice cambios profundos en el modelo educativo chileno (post, perfil de la FEUC, 17 de diciembre), cuestionando que "el programa de la nueva presidenta no representa al movimiento estudiantil" (post, perfil de la FECH, 16 de diciembre). En consecuencia, se llama a la continuidad de la acción colectiva como única garantía para la transformación de la educación, pues "para los próximos cuatro años no habrán reformas posibles sin la participación activa y directa de los movimientos sociales” (De- 
claración de la Confech, perfil de la FECH, 13 de diciembre), por lo que se anuncia "un 2014 lleno de luchas y los estudiantes no bajaremos la voz" (post, perfil de la FECH, 16 de diciembre).

Entre los posts asociados a otros temas, en algunos casos se demanda una participación basada en la acción colectiva frente a unas circunstancias de los mecanismos tradicionales de participación política electoral _-Nunca más sin nosotros" (post, perfil de la FECH, 17 de octubre) — o se convoca a eventos para analizar comunicativamente la campaña electoral (post, perfil de la Feusach, 6 de noviembre).

De manera relevante, algunos comentarios -incluso a posts que no están relacionados con la campaña electoral— hacen referencia al tema. Por ejemplo, se critica a los exlíderes del movimiento que se presentan como candidatos a diputados, denunciando que hayan utilizado con fines personales a los "estudiantes movilizados que entregaron su año académico [en 2011] por ellos y su justa causa" (comentario, perfil de la FECH, 4 de noviembre). Se observa, no obstante, un posicionamiento en los comentarios más crítico con la candidatura de aquellos exdirigentes estudiantiles que formaban parte de la coalición de la Nueva Mayoría —en particular, la figura de Camila Vallejo, en comparación con otros exlíderes con candidaturas independientes, como Gabriel Boric o Francisco Figueroa.

También se observa una cierta contradicción entre la autonomía asumida por las federaciones y la posición personal de algunos usuarios a través de sus comentarios. Frente al posicionamiento de las organizaciones estudiantiles, otros asumen una postura favorable a alguno de los candidatos, señalando que "no votar a Bachelet será un error político de la federación [FECH]" (comentario, perfil de la FECH, 30 de octubre) o que "ahora es cuando podemos cambiar Chile, ¡Vota Marcel Claude!"” (comentario, perfil de la FECH, 18 de octubre).

En particular, en los comentarios de los usuarios, es posible identificar una tensión no resuelta entre la acción colectiva y el posicionamiento por el

5 Candidato presidencial del Movimiento Todos a la Moneda. 
no voto, asumido explícitamente por algunos de los voceros de las federaciones. Los comentarios señalan que si "marchaste por la educación, [... ahora es cuando podemos cambiar Chile" (comentario, perfil de la FECH, 18 de octubre). Se subraya que "no votar significa simplemente darle todo el poder al duopolio. Los movimientos políticos serios, responsables y revolucionarios deben siempre tener vocación de PODER" (comentario, perfil de la FECH, 8 de noviembre), señalando que "no basta con las movilizaciones, si no lo reflejan en las votaciones" (comentario, perfil de la FECH, 13 de noviembre). Así, se propone una estrategia dual - acción colectiva y también participación en la política institucional—, pues "al statu quo hay que atacarlo en su juego y con presión social, $[. .$.$] hay que derrotarlos en$ su juego infiltrando a los nuestros y a la vez presionándolos por fuera" (comentario, perfil de la FECH, 12 de noviembre).

En ese debate generado en los comentarios, se llega a establecer una continuidad entre la baja participación en las votaciones internas de las federaciones y el llamado a no votar en las elecciones presidenciales realizado por las organizaciones estudiantiles: "No es muy sorprendente que haya tan poca participación [en las elecciones estudiantiles] si los mismos ganadores de esta elección, llaman a NO VOTAR" (comentario, perfil de la $\mathrm{FECH}, 13$ de noviembre). Se señala que quienes han asumido la postura de no votar, "más temprano que tarde cambiarán de opinión", pues "la teoría de porqué no lo hacen tiene fallas de fábrica”, señalando la propia "inconsistencia” de que los representantes de los estudiantes se elijan mediante votación (comentario, perfil de la FECH, 14 de noviembre).

Resulta significativo que este debate no se plantee en los perfiles desde las organizaciones estudiantiles, sino que emerja en los comentarios de los usuarios, de manera espontánea, lo cual confirma el criterio antes mencionado de los entrevistados sobre las características del uso de estos recursos tecnológicos. Ello muestra la complejidad de la cuestión y la falta de consenso al respecto y que Facebook no se visibiliza como un espacio para este debate por parte de las federaciones estudiantiles en el contexto de la campaña electoral, sino que se asocia más a la acción colectiva y a los procesos organizativos internos, en correspondencia con las particularidades de un movimiento social que aparece mediado por organizaciones estudiantiles de carácter tradicional. 


\section{Conclusiones}

El análisis muestra que, en un contexto caracterizado por la baja movilización social e importantes procesos organizativos internos, el uso de las redes sociales por parte de las organizaciones que forman parte del movimiento se centra principalmente en las funciones informativa - como fuente de información y espacio para compartir contenidos- y organizativa - en relación con los procesos internos, como recursos para la divulgación de convocatorias y actividades vinculadas-. Se reiteran otros usos también identificados en estudios previos ya mencionados, como destacar logros - en este caso, la influencia de las demandas del movimiento en la agenda electoral - y señalar a sus oponentes —en la campaña electoral, estos se asocian a los partidos políticos y candidatos que, se considera, intentan la cooptación real o simbólica del movimiento-. También se confirma la tendencia identificada por tales estudios a un incremento en la actividad en las redes sociales durante los momentos de mayor movilización, aun cuando esta no se halle vinculada a la campaña electoral.

Tales tendencias son coherentes con una baja tasa de interactividad observada, cuyos niveles más elevados estarían asociados a otro tipo de usos de las redes sociales digitales, por ejemplo, como espacios de debate. Tal uso resulta contradictorio tanto con las particularidades de estas plataformas de la Web 2.0, en cuya caracterización se suele destacar precisamente su naturaleza bidireccional, facilitadora del debate, como con su uso específico en un contexto comunicacionalmente tan relevante para el debate público como es una campaña electoral. Esta baja tasa de interactividad tiene su correlato, precisamente, en el bajo uso de Facebook focalizado en la campaña electoral, en cuanto recurso de visibilización autónoma para mediar las agendas políticas de la campaña. En el caso específico de los posts vinculados a este tema, las diferencias en el comportamiento de las tasas de interactividad podría estar asociado a la mediación ejercida por las peculiaridades de cada federación estudiantil, y de sus respectivas universidades, si bien sería necesaria una indagación posterior más profunda al respecto para afirmarlo.

En los usos focalizados en la campaña electoral, el análisis muestra una tensión entre el nivel institucional y el individual. El primero prioriza 
el silencio en torno al tema, como estrategia para salvaguardar la autonomía del movimiento frente a los partidos políticos, en un momento de especial densidad de la comunicación política institucional que supone un elevado riesgo de cooptación comunicativa del movimiento, incrementado por el tránsito a la política de exdirigentes. En el segundo, los sujetos sí proponen unos usos de las redes sociales como espacios para apoyar o rechazar candidatos, compartir creencias políticas, criticar determinados posicionamientos político-ideológicos e interactuar en sentido general sobre temas políticos, tal como estudios precedentes han mostrado. Esta tensión entre el uso colectivo organizado y el individual en la acción colectiva muestra la complejidad y conflictividad inherente a los usos de las redes sociales por parte de la acción colectiva en contextos de una importante comunicación política institucional.

Del análisis, además, se puede concluir que hay varias mediaciones relevantes para los usos de las redes sociales por parte del movimiento social en el contexto de la campaña electoral. En primer lugar, la tensión entre lo social y lo político y la perspectiva que se asume desde las organizaciones estudiantiles y sus líderes, en particular los que se posicionan desde una defensa radical de la autonomía del movimiento y una comprensión de la disputa del poder que no está asociada a la democracia representativa y, por tanto, a las elecciones. En un escenario de campaña electoral, se considera mayor la posibilidad de cooptación (orgánica o simbólica) del movimiento por la política institucional, complejizando el papel comunicativo del movimiento, reforzado por la presencia de organizaciones más tradicionales en su interior y el tránsito de antiguos líderes hacia la política institucional.

Esta mediación conecta con la tensión entre lo institucional y lo individual en el uso de la red social, pues, mientras hay un distanciamiento explícito de la acción colectiva organizada (federaciones) en relación con las candidaturas tanto presidenciales como de sus exlíderes candidatos a diputados, el tema está presente en los comentarios de los usuarios. La ambigüedad conflictiva de la relación del movimiento social con la política institucional reforzaría cierta incertidumbre en el papel del movimiento como mediación comunicativa en la campaña y, en consecuencia, acerca de los usos de las redes sociales. 
En segundo lugar, otra mediación relevante es la tensión entre acción colectiva en línea y fuera de línea. La mayor actividad en las redes sociales durante las movilizaciones en comparación con los momentos más significativos de la campaña electoral, como los debates entre candidatos, confirma la continuidad de la estrecha articulación entre acción colectiva en línea (a través de las redes sociales) y acción colectiva fuera de línea (movilizaciones, protestas), que resulta más importante que el uso de la red social como espacio de debate en torno a la campaña electoral. Finalmente, en esta tensión, problematizada por estudios previos sobre los usos de las redes sociales como parte de la acción colectiva, se añade otra mediación: las particularidades del movimiento social y el momento específico en que se encuentra. Así, la presencia de organizaciones de carácter tradicional en el movimiento social (las federaciones estudiantiles) favorece una cierta prioridad de la dimensión organizativa y los procesos internos de las organizaciones en los usos de los perfiles de Facebook, incluso en un escenario tan importante para la visibilización autónoma y el debate público, como es la campaña electoral.

\section{Referencias}

Anduiza, E., Cristancho, C. y Sabucedo, J. M. (2014). Mobilization through online social networks: the political protest of the indignados in Spain. Information, Communication \& Society, 17(6), 750-764. doi: 10.1080/1369118X.2013.808360

Bennet, W. L. y Segerberg, A. (2012). The logic of connective action: digital media and the personalization of contentious politics. Information, Communication \& Society, 15(5), 739-768. doi: 10.1080/1369118X.2012.670661

Cabalín-Quijada, C. (2014). Estudiantes conectados y movilizados: el uso de Facebook en las protestas estudiantiles en Chile. Comunicar, 43, 25-33. doi: 10.3916/C43-2014-02

Castells, M. (2009). Comunicación y poder. Madrid: Alianza Editorial. 
Castells, M. (2012). Redes de indignación y esperanza: los movimientos sociales en la era de internet. Madrid: Alianza Editorial.

Castells, M. (2014a). El poder de las redes. Vanguardia Dossier, 50, 6-13.

Castells, M. (2014b). El impacto de internet en la sociedad: una perspectiva global. En VV.AA, C@mbio: 19 ensayos clave acerca de cómo internet está cambiando nuestras vidas (pp. 127-148). Madrid: OpenMind-BBVA.

Castillo Esparcia, A., García Ponce, D. y Smolak Lozano, E. (2013). Movimientos sociales y estrategias de comunicación: el caso del $15 \mathrm{My}$ de Occupy Wall Street. Estudios sobre el Mensaje Periodístico, 19(1), 71-89. doi: 10.5209/rev_ESMP.2013.v19.n1.42508

Cerrillo Garnica, O. y Lay Arellano, I. T. (2014). \#Yosoy132: redes digitales como comunicación e identidad en la acción colectiva. Enfoques, 13(1), 294-317.

D'heer, E. y Verdegem, P. (2014). Conversations about the elections on Twitter: towards a structural understanding of Twitter's relation with the political and the media field. European Journal of Communication, 29(6), 720-734. doi:10.1177/0267323114544866

Dahlberg, L. y Siapera, E. (eds.) (2007). Radical democracy and the internet: interrogating theory and practice. Basingstoke: Palgrave Macmillan.

Davis, S., Elin, L. y Reeher, G. (2002). Click on democracy: the Internet's power to change political apathy into civic action. Boulder, CO: Westview Press.

Duggan, M., Ellison, N. B, Lampe, C., Lenhart, A. y Madden, M. (2014). Demographics of key social networking platforms. Recuperado de http://www.pewinternet.org/2015/01/09/demographics-of-keysocial-networking-platforms-2/ 
Escandón Montenegro, P. (2013). Política electoral y política ciudadana: partidos, movimientos sociales y anticandidatos en la campaña preelectoral del Ecuador. Razón y Palabra, 82, 24-14.

Fairclough, N. (1995). Critical discourse analysis: the critical study of language. Harlow, UK: Longman.

Fairclough, N. (2003). Analyzing discourse: textual analysis for social research. Nueva York: Routledge.

Fernández-Planells, A., Feixa Pampols, C. y Figueroas-Maz, M. (2013). 15-M en España: diferencias y similitudes en las prácticas comunicativas con los movimientos previos. Última Década, 21(39), 115-138. doi:10.4067/S0718-22362013000200006

Franzosi, R. (1998). Narrative analysis -Or why (and how) sociologists should be interested in narrative. Annual Review of Sociology, 24, 517-554. doi: 10.1146/annurev.soc.24.1.517

Gómez García, R. y Treré, E. (2014). The \#YoSoy132 movement and the struggle for media democratisation in Mexico. Convergence: The International Journal of Research into New Media Technologies 1354856514541744, July 20, 2014. doi: $10.1177 / 1354856514541744$

Guzmán-Concha, C. (2012). The sudents' rebellion in Chile: occypy protest or classical social movement? Social Movements Studies: Journal of Social, Cultural and Political Protest, 11(3-4), 408-415. doi: 10.1080/14742837.2012.710748

Herring, S. (2004). Computer-mediated discourse analysis: an approach to researching online behavior. En Barab, S. Kling, R. y Gray, J. (eds.), Designing for virtual communities in the service of learning (pp. 338376). Nueva York: Cambridge University Press. 
Juris, J. S. (2012). Reflections on \# occupy everywhere: social media, public space, and emerging logics of aggregation. American Ethnologist, 39, 259-279. doi: 10.1111/j.1548-1425.2012.01362.x

Larsson, A. O. y Moe, H. (2011). Studying political microblogging: Twitter users in the 2010 Swedish election campaign. New Media \& Society, 14(5), 729-747. doi:10.1177/1461444811422894

Melucci, A. (2004). The process of collective identity. En Jonhston, H. y Klandermans, B. (eds.), Social movements and culture (pp. 41-63). Minneapolis, MN: University of Minnesota Press.

Offe, C. (1988). Partidos políticos y nuevos movimientos sociales. Madrid: Sistema.

Panagopoulos, C., Gueorguieva, V., Slotnick, A., Gulati, G. y Williams, C. (2009). Politicking online: the transformation of election campaign communications. Newark, NJ: Rutgers University Press.

Paz, F. (2014). De la arena social a la arena política: un análisis sobre el movimiento estudiantil chileno. Cuadernos del Ciesal, 11(13), 101-124.

Routledge, P. (1997). A spatiality of resistance: theory and practice in Nepal's revolution of 1990. En Keith, M. y Pile, S. (eds.), Geographies of resistance (pp. 68-86). Londres: Routledge.

Sarıtaş, A. y Aydın, E. E. (2015). Elections and social media: an overview. International Journal of Social Ecology and Sustainable Development (IJSESD), 6(1), 59-72. doi:10.4018/ijsesd.2015010105

Taylor, V. y Whittier, N. (2004). Analytical approaches to social movements culture: the culture of the women's movement. En Jonhston, $\mathrm{H}$. y Klandermans, B. (eds.), Social movements and culture (pp. 163187). Minneapolis, MN: University of Minnesota Press. 
Túñez López, M. (2012). Los periódicos en las redes sociales: audiencias, contenido, interactividad y estrategias comerciales. Estudios sobre el Mensaje Periodístico, 18(1), 221-239. doi:10.5209/rev ESMP.2012.v18.n1.39367

Valenzuela, S., Arriagada, A.y Scherman, A. (2012). The social media basis of youth protest behavior: the case of Chile. Journal of Communication, 62(2), 299-314. doi: 10.1111/j.1460-2466.2012.01635.x

Vitak, J., Zube, P., Smock, A., Carr, C. T., Ellison, N. y Lampe, C. (2011). It's complicated: Facebook users' political participation in the 2008 election. Cyberpsychology, Behavior, and Social Networking, 14(3), 107-114. doi:10.1089/cyber.2009.0226

Walgrave, S. y Van Aelst, P. (2006). The contingency of the mass media's political agenda setting power: toward a preliminary theory. Journal of Communication, 56(1), 88-109.

Woolley, J. K., Limperos, A. M. y Oliver, M. B. (2010). The 2008 Presidential Election, 2.0: a content analysis of user-generated political Facebook groups. Mass Communication and Society, 13(5), 631-652. doi: $10.1080 / 15205436.2010 .516864$

Zibechi, R. (2004). El otro mundo es el "adentro" de los movimientos sociales. América Latina en Movimiento, 386, 49-52.

Zonta Díaz, N. (2013). Interactividad de las cadenas autonómicas de televisión en las redes sociales: estudio de caso y propuesta de proyecto de comunicación para RTVCyL en Facebook (Tesis inédita de maestría, Universidad de Valladolid, España). Recuperado de http://uvadoc.uva.es/handle/10324/3782 